\title{
Highlighting the road towards new disease-specific comorbidity indices
}

\author{
Paolo Dell'Oglio ${ }^{1}$, Elio Mazzone ${ }^{2}$, Zhe Tian $^{3}$, Pierre I. Karakiewicz ${ }^{3,4}$ \\ ${ }^{1}$ Department of Urology, ASST Grande Ospedale Metropolitano Niguarda, Milan, Italy; ${ }^{2}$ Division of Oncology/Unit of Urology, Urological \\ Research Institute, IRCCS Ospedale San Raffaele, Milan, Italy; ${ }^{3}$ Cancer Prognostics and Health Outcomes Unit, University of Montreal Health \\ Center, Montreal, Canada; ${ }^{4}$ Department of Urology, University of Montreal Health Center, Montreal, Canada \\ Correspondence to: Paolo Dell'Oglio, MD. Department of Urology, ASST Grande Ospedale Metropolitano Niguarda, Milan, Italy. \\ Email: paolo.delloglio@gmail.com. \\ Provenance and Peer Review: This article was commissioned by the editorial office, Translational Andrology and Urology. The article did not undergo \\ external peer review. \\ Response to: Fonteyne V, Rammant E, Decaestecker K. Predicting perioperative mortality after radical cystectomy: comorbidity assessment tools are \\ only part of the puzzle. Transl Androl Urol 2019;8:781-4; \\ Regmi SK, Konety BR. Studying the impact of comorbidity on post radical cystectomy survival: have we come a full circle? Transl Androl Urol \\ 2019;8:S314-7.
}

Submitted Mar 27, 2020. Accepted for publication May 11, 2020.

doi: $10.21037 /$ tau-20-832

View this article at: http://dx.doi.org/10.21037/tau-20-832

We thank Regmi et al. (1) and Fonteyne et al. (2) for their comments on our recently published article in Annals of Surgical Oncology (3). As noted by Dr. Fonteyne (2), the key point of our study is that, differently from all the current available comorbidity indices (4), our new comorbidity assessment tool was developed and validated, in a cohort of respectively $\sim 6,000$ and 1,000 muscle invasive bladder cancer (BCa) patients treated with radical cystectomy (RC), for prediction of 90-day mortality. Therefore, it was specifically designed for RC patients. In this context, it is important to emphasize that comorbidity profiles differ depending on the primary underlying pathology of interest, here BCa. The Charlson comorbidity index (CCI) (5), that is the most used and supported by urological guidelines for retrospective quantification of comorbid conditions $(4,6)$, was developed in 1987 using a sample of hospitalized medical patients. As such, it is not tailored for RC patients. Moreover, it relies on several comorbid conditions that are rare or even non applicable to contemporary RC patients, such as connective tissue disease, moderate or severe liver disease, dementia, leukemia, lymphoma, metastatic solid tumours and AIDS $(3,7,8)$. Furthermore, other conditions, such as BCa manifestations, are not included in the CCI, even though they are specific to and apply in to contemporary RC patients $(3,8)$. These observations strongly suggest that, if we rely on the original CCI (5), we cannot properly quantify the comorbidity profile of contemporary RC patients. As a consequence, improper classification of comorbidity profiles cannot result in valid application of the outdated CCI in contemporary analyses of RC studies. Indeed, when we analyzed all possible baseline comorbid conditions and BCa manifestations using 5,920 ICD-9-CM diagnostic codes relying on highly robust statistical analyses (i.e., simulated annealing) (3), we identified a group of 6 individual comorbid conditions and 2 BCa manifestations that satisfied the criteria of maximal accuracy and parsimony for prediction of 90-day mortality after RC (Figure 1), after accounting for patient (i.e., age, gender, race) and tumour characteristics (i.e., pathological $\mathrm{T}$ stage and $\mathrm{N}$ stage). Only two of these conditions were accounted within the original CCI, namely chronic obstructive pulmonary disease and congestive heart failure. It is also of note that $2 \mathrm{BCa}$ manifestations (i.e., urinary tract infection-UTI and hydronephrosis) were also included in the final model, suggesting that comorbidities should not be the sole determinant for predicting perioperative mortality after RC, as outlined by Regmi et al. (1) and Fonteyne et al. (2). To the best of our knowledge, we are the 


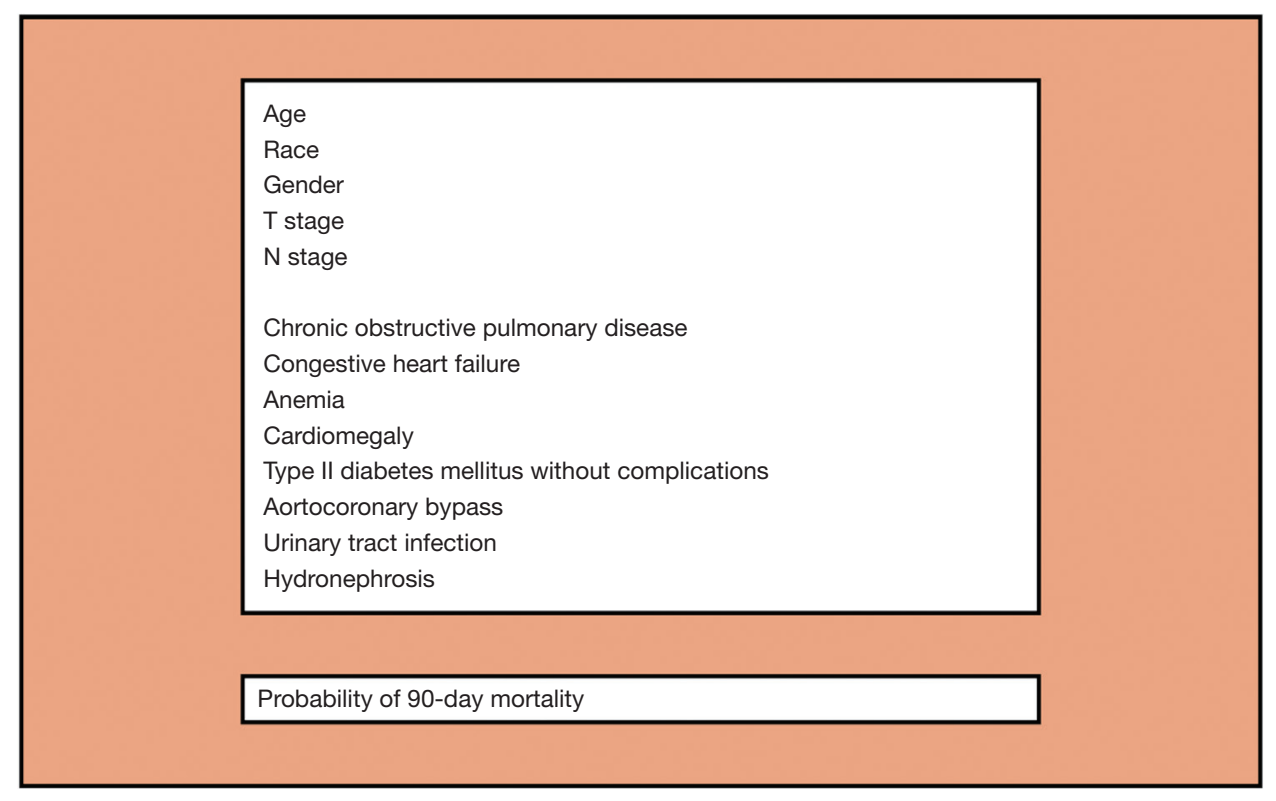

Figure 1 Risk calculator based on the combination of 6 individual comorbid conditions and 2 individual bladder cancer manifestations that allows individual estimate of 90-day mortality after radical cystectomy (3).

first to introduce the presence of disease manifestations within a model framework. It indicates that their documentation prior to RC is crucial. Of note, hydronephrosis is known to be associated with aggressive disease and lower survival rates after RC (9). Conversely, for the first time, UTI recorded prior to $\mathrm{RC}$ was demonstrated to represent a risk factor for 90-day mortality after surgery. Regmi et al. (1) and Fonteyne et al. (2) accurately stated that other factors, such as frailty and sarcopenia, that are both well-recognized elements for increased risk of mortality after RC (4), "should be part of the puzzle" to predict perioperative mortality after RC and therefore should be included into our model. However, despite being considered in our statistical analyses (>5,900 ICD-9-CM diagnostic codes were assessed), they failed to make the cut into the final tool. Nevertheless, future studies should validate or reject the need for their consideration in RC-specific comorbidity assessments.

Furthermore, in their comment, Regmi and colleagues (1) questioned the ability of ICD-9-CM diagnostic codes to capture all relevant comorbidities and commented on the high mortality rate of our cohort, which might have influenced our results (i.e., $10.4 \%$ and $13.1 \%$ in the development and validation cohort, respectively). Despite the latter is located within the upper perioperative mortality rate range of RC patients (2-13\%) (4), we agree that ICD-9-CM diagnostic codes might not capture all comorbidities. However, they are generally considered a reliable method (10). To ensure that all relevant codes were included in our analyses, we calculated the frequency of all possible individual diagnostic codes that appeared in the database by classifying inpatient, outpatient claims and physician billing claims, up to 12 months prior RC (3). This methodology added to the list of codes. Our concern in that regard is based on potential under-reporting of BCa manifestations (such as UTI and hydronephrosis) in discharge records and consequently in databases that rely on discharge records. However, we also believe that the large size of population-based studies allows to compensate for some of such limitations and provides accurate assessment of "real world" outcomes, ensuring broad generalizability of findings.

Regmi et al. (1) also pointed out that we did not provide comparative statistics between the increase in accuracy of our tool relative to the original CCI (AUC 71.1\% vs. 68\%). In response to this pertinent comment, we refer to the European guidelines for reporting statistics in urology (11) which do not recommend to add $\mathrm{p}$-values for differences in discrimination between two models. This said, even if there is still margin to improve the predictive ability of the model, this difference in accuracy suggests that our model performs better than the original CCI and it is also simpler requiring only 8 variables for individual 90-day 
mortality risk estimation (Figure 1). Another strength of our manuscript that should be pointed out is that this new developed risk tool (Figure 1) does not rely on artificial weights of comorbidities as the original CCI (5), that might attribute incorrect importance to one or more comorbid conditions. Specifically, in our model, the impact of each comorbid conditions on 90-day mortality after surgery was defined using logistic regression derived coefficients.

To conclude, our study represents the first attempt to develop a contemporary comorbidity tool specific for RC patients as recommended by several authorities $(4,6,7)$. Despite its strengths, our model is not devoid of important limitations. Indeed, it was devised using administrative data that rely on ICD-9-CM diagnostic codes and on patients aged 66 years or older. Although, we could argue that two third of $\mathrm{RC}$ candidates are older than 60 (4), our tool is not applicable to younger patients. Moreover, as correctly underlined by Fontayne and colleagues (2), we were not able to take into account other key factors, such as nutritional status, prehabilitation programs, hospital annual surgical volume and smoking exposure (12). All these might impact postoperative mortality after RC. Therefore, we agree with the authors that there is still work to be done in order to increase the accuracy of our model and to implement it in daily clinical practice. However, researchers should take advantage of our study and its methodology to promote diseasespecific comorbidity indices in order to improve patient counselling and provide accurate risk-adapted strategies. "What you measure depends on the tool you use" (13) and contemporary disease specific comorbidity tools are mandatory to optimally characterize patients candidate for a specific treatment and to avoid to rely on unprecise data.

\section{Acknowledgments}

Funding: None.

\section{Footnote}

Conflicts of Interest: The authors have completed the ICMJE uniform disclosure form (available at http://dx.doi. org/10.21037/tau-20-832). The authors have no conflicts of interests to declare.

Ethical Statement: The authors are accountable for all aspects of the work in ensuring that questions related to the accuracy or integrity of any part of the work are appropriately investigated and resolved.

Open Access Statement: This is an Open Access article distributed in accordance with the Creative Commons Attribution-NonCommercial-NoDerivs 4.0 International License (CC BY-NC-ND 4.0), which permits the noncommercial replication and distribution of the article with the strict proviso that no changes or edits are made and the original work is properly cited (including links to both the formal publication through the relevant DOI and the license). See: https://creativecommons.org/licenses/by-nc-nd/4.0/.

\section{References}

1. Regmi SK, Konety BR. Studying the impact of comorbidity on post radical cystectomy survival: have we come a full circle? Transl Androl Urol 2019;8:S314-7.

2. Fonteyne V, Rammant E, Decaestecker K. Predicting perioperative mortality after radical cystectomy: comorbidity assessment tools are only part of the puzzle. Transl Androl Urol 2019;8:781-4.

3. Dell'Oglio P, Tian Z, Leyh-Bannurah SR, et al. Development of a New Comorbidity Assessment Tool for Specific Prediction of Perioperative Mortality in Contemporary Patients Treated with Radical Cystectomy. Ann Surg Oncol 2019;26:1942-9.

4. Williams SB, Kamat AM, Chamie K, et al. Systematic Review of Comorbidity and Competing-risks Assessments for Bladder Cancer Patients. Eur Urol Oncol 2018;1:91-100.

5. Charlson ME, Pompei P, Ales KL, et al. A new method of classifying prognostic comorbidity in longitudinal studies: development and validation. J Chronic Dis 1987;40:373-83.

6. Guidelines on muscle-invasive and metastatic bladder cancer. European Association of Urology. 2019.

7. Dell'Oglio P, Tian Z, Leyh-Bannurah SR, et al. ShortForm Charlson Comorbidity Index for Assessment of Perioperative Mortality After Radical Cystectomy. J Natl Compr Canc Netw 2017;15:327-33.

8. Lambert E, D'Hondt F, Mazzone E, et al. Time to Move On: The Impending Need for a New Diseasespecific Comorbidity Index for Bladder Cancer Patients Undergoing Robot-assisted Radical Cystectomy. Eur Urol Focus 2019. doi: 10.1016/j.euf.2019.05.015.

9. Oh JJ, Byun SS, Jeong CW, et al. Association Between Preoperative Hydronephrosis and Prognosis After Radical Cystectomy Among Patients With Bladder Cancer: A Systemic Review and Meta-Analysis. Front Oncol 
2019;9:158.

10. Deyo RA, Cherkin DC, Ciol MA. Adapting a clinical comorbidity index for use with ICD-9-CM administrative databases. J Clin Epidemiol 1992;45:613-9.

11. Vickers AJ, Sjoberg DD, European U. Guidelines for reporting of statistics in European Urology. Eur Urol 2015;67:181-7.

12. Cacciamani GE, Ghodoussipour S, Mari A, et al.

Cite this article as: Dell'Oglio P, Mazzone E, Tian Z, Karakiewicz PI. Highlighting the road towards new diseasespecific comorbidity indices. Transl Androl Urol 2020;9(3):14751478. doi: $10.21037 /$ tau-20-832
Association Between Smoking Exposure, Neoadjuvant Chemotherapy Response and Survival Outcomes Following Radical Cystectomy: Systematic Review and Meta-Analysis. J Urol 2020. doi: 10.1097/ JU.0000000000000813.

13. Artibani W. What You Measure Depends on the Tool You Use: A Short Step from Incorrect Measurements to Fake Data. Eur Urol 2018;74:8-9. 